\title{
Responses of Viscerosomatic VPL Neurons to Intracardiac Bradykinin
}

\author{
Hajime Horie and Toshikatsu Yokota \\ Department of Physiology, Medical College \\ of Shiga, Otsu, Japan
}

(Received 22 April 1991)

\begin{abstract}
Responses of VPL neurons to intracardiac injection of bradykinin $(2 \mu \mathrm{g} / \mathrm{kg})$ were examined in urethane-chloralose-anesthetized cats. A total of $39 \mathrm{VPL}$ neurons were subjected to the study. Nociceptive specific (NS) and wide dynamic range (WDR) neurons receiving both cutaneous nociceptive and cardiac sympathetic afferent inputs regularly exhibited an increased discharge in response to intracardiac bradykinin. NS neurons responsive to intracardiac bradykinin had a circumscribed cutaneous receptive field in the area corresponding to tactile dermatomes $\mathrm{C}_{5}-\mathrm{T}_{13}$. WDR neurons responsive to intracardiac bradykinin had at least a part of their receptive fields in the same area. In contrast, NS and WDR neurons unresponsive to cardiac sympathetic afferents, and low threshold mechanoreceptive (LTM) neurons having a circumscribed receptive field in $\mathrm{C}_{5}-\mathrm{T}_{13}$ dermatomes did not respond to intracardiac bradykinin. Both NS and WDR neurons responsive to intracardiac bradykinin were located in the shell region of the medial part of caudal VPL. These cutaneous nociceptive neurons may be involved in transmitting cardiac nociceptive information to the cerebral cortex in the cat.
\end{abstract}

Key Words : bradykinin, thalamus, cardiac pain, nociception, nucleus ventralis posterolateralis, cat

\section{INTRODUCTION}

Clinical and experimental evidence has demonstrated that the sympathetic cardiac afferent fibers are essential for the conduction of pain originating in the heart. For example, an- ginal pain in man ${ }^{11,12,21,22)}$ and pseudoaffective reactions accompanying coronary artery occlusion in experimental animals ${ }^{5,14}$ can be obliterated by cervicothoracic sympathetic ganglionectomy. In animal studies, increased firing in both $\mathrm{A} \delta$ and $\mathrm{C}$ cardiac sympathetic afferent 
fibers could be evoked by myocardial ischemia ${ }^{6,17,19)}$. Electrophysiological experiments have demonstrated that sympathetic cardiac afferent fibers project to neurons in the spinal $\operatorname{cord}^{3,7,8,16)}$, which also receive convergent nociceptive somatic inputs. Such observations of viscerosomatic integration have provided one substrate for referred pain of cardiac origin. Previously, we reported that a significant proportion of cutaneous nociceptive neurons in the shell region of the caudal part of the nucleus ventralis posterolateralis (VPL) of the cat thalamus responded to electrical stimulation of sympathetic afferent fibers in the inferior cardiac nerve (ICN) ${ }^{15}$. These results suggest that the shell region of the caudal VPL constitutes a thalamic link in a cardiac pain pathway, and that cardiac and cutaneous pain systems share a common projection locus in the VPL. It the present study, it was attempted to confirm these previous observations using intracardiac injection of a natural algogenic substance, bradykinin.

\section{METHODS}

Thirty four cats weighing $2.9 \sim 5.6 \mathrm{~kg}$ were tranquilized with intramuscular ketamine $(20 \mathrm{mg} / \mathrm{kg})$, and were then anesthetized with 3.5 $\mathrm{m} l / \mathrm{kg}$ of urethane-chloralose solution (urethane $125 \mathrm{mg} / \mathrm{ml}$, chloralose $10 \mathrm{mg} / \mathrm{ml}$ ). Throughout the recording procedure, the cats were paralyzed with intravenous pancuronium and supplemental doses of urethane-chloralose solution were administered as required. The animals were artificially ventilated to maintain expiratory $\mathrm{CO}_{2}$ between 3.5 and $4.5 \%$. Each cat's body temperature was monitored with an esophageal probe, and core temperature was maintained at $37 \pm$ $1^{\circ} \mathrm{C}$. A catheter for recording arterial blood pressure was implanted in the femoral artery. A left thoracotomy in the second intercostal space provided and opening for placement of a bipolar stimulating electrode on the inferior cardiac nerve. The electrode was held in place with low melting point wax. For intracardiac injection of bradykinin, a canula was inserted into the right atrium. After the animals were mounted in a stereotaxic apparatus, a craniotomy was performed to allow insertion of recording microelectrodes into the right or left VPL. The recording microelectrodes were filled with $2 \%$ pontamine sky blue in $1 \mathrm{M}$ sodium acetate. The VPL was explored for neurons receiving cutaneous nociceptive input. Once a cutaneous nociceptive neuron was identified, responses to electrical stimulation of the ICN were examined. If the neuron responded to the stimulation, $2 \mu \mathrm{g} /$ $\mathrm{kg}$ of bradykinin was injected into the right atrium, and neuron's response was recorded. The output of the oscilloscope on which the activities of single thalamic neurons were displayed, was connected to a window discriminator, which was itself connected to a spike counter. The output from the spike counter consisted of a count of the number of spikes occurring in each sequential $1-\mathrm{s}$ bin. The firing rates and window discriminator pulses were recorded on a chart recorder. After concluding an observation of interest, the recording site was marked with pontamine sky blue, extruded from the tip of the microelectrode by passing a current of $5 \mu \mathrm{A}$ (electrode negative) through it for $10 \mathrm{~min}$. At the termination of each experiment, the brain was perfused with $1,000 \mathrm{~m} l$ of normal saline followed by $3,000 \mathrm{~m} l$ of $10 \%$ formalin-saline. After postfixation for at least 1 week, the brain was frozen, cut into $50 \mu \mathrm{m}$ coronal sections, and stained with cresyl-violet. Dye marks were identified in the stained sections.

\section{RESULTS}

A total of 27 cutaneous nociceptive VPL neurons receiving cardiac sympathetic afferent input were tested for responses to intracardiac injection of bradykinin. Twenty of them were 
Fig. 1 A NS neuron responsive to intracardiac bradykinin. A : cutaneous receptive field (marked by a black area). B : raster dot display of neuron's responses to double shock stimulation (indicated by two arrows) of the ICN. C: responses of the neuron to brushing, pressure and noxious pinch applied to the cutaneous receptive field. Upper trace shows rate of neuronal discharge in impulses per s. Lower trace shows output of window discriminator. Each deflection represents one neuronal potential. Horizontal bars indicate periods of stimulus application. Duration of each stimulus was 10s. D : responses of the neuron to a bolus dose of $2 \mu \mathrm{g} / \mathrm{kg}$ bradykinin into the right atrium. Blood pressure record is shown together with rate of neuronal discharge and output of window discriminator.

Fig. 2 A WDR neuron responsive to intracardiac bradykinin. A : cutaneous receptive field. In black area, the neuron had a graded response to brushing, pressure and noxious pinch. In cross-hatched area, the neuron did not respond to brushing, but differentially responded to pressure and noxious pinch. In shaded area, the neuron exclusively responded to noxious pinch. B : raster dot display of responses to electrical stimulation of the ICN. C : responses of the neuron to mechanical stimulation of 3 different areas of the cutaneous receptive field. Traces $a, b$ and $c$ represent responses to stimulation of corresponding areas indicated by arrows in $\mathrm{A}$. Horizontal bars indicate periods of stimulus application. D : responses of the neuron to a bolus dose of $2 \mu \mathrm{g} / \mathrm{kg}$ bradykinin into the right atrium.
A
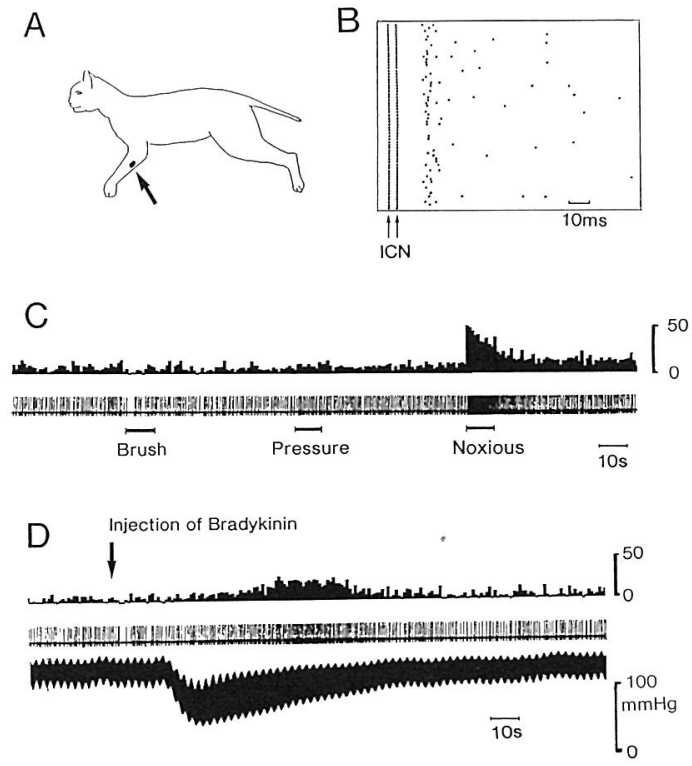

A

B
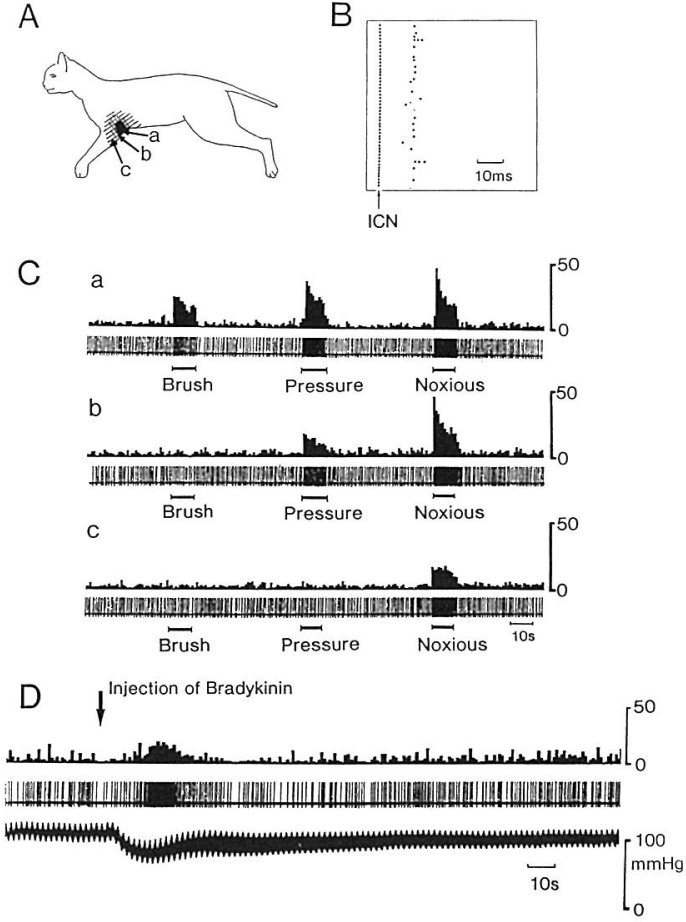
nociceptive specific (NS) neurons. They were unresponsive to weak forms of mechanical stimulation such as brushing, but discharged when intense mechanical stimuli were applied to a circumscribed contralateral cutaneous area located in the forelimb or upper trunk (black area in Fig. 1-A). The remaining 7 neurons were wide dynamic range (WDR) neurons. They had a graded response to brushing, pressure and noxious pinch applied to the center of the receptive field (black area in Fig. 2-A), and responded best to noxious pinch. The center was located in the contralateral forelimb or upper trunk. Outside this zone (cross-hatched area in Fig. 2-A), they were unresponsive to low-intensity mechanical stimuli, but responded differentially to pressure and noxious pinch. Finally, the latter area was surrounded by an area in which only noxious pinch resulted in neuronal discharges (shaded area in Fig. 2-A). The receptive field was confined to the contralateral half of the body surface. Both NS and WDR neurons of this group responded to single or double shock stimulation of the ICN with latencies $10.0 \sim 18.4 \mathrm{~ms}$ $(12.4 \pm 2.4 \mathrm{~ms})$ and $11.3 \sim 14.8 \mathrm{~ms}(12.0 \pm 1.2 \mathrm{~ms})$, respectively.

When $2 \mu \mathrm{g} / \mathrm{kg}$ of bradykinin was injected into the right atrium, all of them exhibited an increase in discharge rate, and the activity was not related to the cardiac cycle. The latency to the onset of increased discharge was $11 \sim 29 \mathrm{~s}$ $(19.6 \pm 4.8 \mathrm{~s})$ in NS neurons, whereas it was 11 $\sim 25$ s $(18.4 \pm 5.8 \mathrm{~s})$ in WDR neurons. The duration of increased discharge was $36 \sim 189 \mathrm{~s}$ (81.1土 $45.3 \mathrm{~s})$ and $32 \sim 151 \mathrm{~s}(68.9 \pm 40.5 \mathrm{~s})$, in NS and WDR neurons, respectively. The discharge rate of NS neurons was $51.3 \pm 28.7$ spikes/10s before the injection. It increased to $188.0 \pm 86.9$ spikes/ $10 \mathrm{~s}$ in response to intracardiac bradykinin. The discharge rate of WDR neurons increased from $51.2 \pm 29.7$ spikes $/ 10$ s to $192.0 \pm 65.4$ spikes $/ 10$ s. The discharge rate increased by $92 \sim 1,472 \%$
$(464 \pm 330 \%)$ in NS neurons, whereas it increased by $143 \sim 1,258 \%(472 \pm 431 \%)$ in WDR neurons. Responses of a NS neuron and of a WDR neuron to intracardiac bradykinin are illustrated in Fig. 1-D and Fig. 2-D, respectively.

NS neurons responsive to intracardiac bradykinin had a circumscribed cutaneous receptive field in the area corresponding to tactile dermatomes $\mathrm{C}_{5}-\mathrm{T}_{13}$. WDR neurons responsive to intracardiac bradykinin had at least a part of their fields in the same area. Both NS and WDR neurons responsive to intracardiac bradykinin were located in the dorsal or ventral shell region of the medial part of caudal VPL, but WDR neurons were located more rostrally than NS neurons were.

For comparison, responses to intracardiac bradykinin were tested in $5 \mathrm{NS}$ and 2 WDR neurons which did not respond to electrical stimulation of the ICN. None of them responded with an increased discharge to the injection. Responses to intracardiac bradykinin were also tested in 5 low threshold mechanoreceptive (LTM) neurons which had a circumscribed cutaneous receptive field either in the forearm (3 neurons) or in the chest wall ( 2 neurons). Their receptive fields were included in $\mathrm{C}_{5}-\mathrm{T}_{13}$ dermatomes. Again none of them exhibited an increased discharge in response to the injection.

\section{DISCUSSION}

Intra-arterial injection of bradykinin in cats and dogs can elicit pseudoaffective response indicative of pain ${ }^{5)}$, Bradykinin levels in coronary sinus increase following coronary artery occlusion $^{10)}$. Bradykinin applied to the heart stimulates $\mathrm{A} \delta$ - and $\mathrm{C}$ afferent fibers originating in the heart and coursing in sympathetic nerves to the spinal cord ${ }^{2,12,13,18)}$. Thus, the possibility exists that noxious cardiac events release bradykinin, which in turn stimulates cardiac sympa- 
thetic afferent fibers coursing toward the spinal cord. It has been demonstrated that application of bradykinin to the heart can excite neurons of the spinothalamic and spinoreticular tracts in the spinal $\operatorname{cord}^{1,4,5)}$. Results of the present experiments indicate that intracardiac injection of bradykinin excites neurons in the shell region of the caudal VPL of the cat thalamus, which receive both cutaneous nociceptive and cardiac sympathetic afferent inputs relayed through the spinal cord. These results support that the shell region of the caudal VPL may be involved in transmitting cardiac nociceptive information to the cerebral cortex in the cat.

\section{ACKNOWLEDGEMENTS}

This work was entrusted to the Department of Physiology, Medical College of Shiga by the Science and Technology Agency, using the Special Coordination Funds for Promoting Science and Technology.

\section{References}

1) Ammons, W. S., Girardot, M.-N. and Foreman, R. D., Effects of intracardiac bradykinin on $\mathrm{T}_{2}-$ $\mathrm{T}_{5}$ medial spinothalamic cells, Amer. J. Physiol., 249 (Regulatory Integrative Comp. Physiol. 18) (1985) R147-R152.

2) Baker, D. G.,Coleridge, H. M., Cleridge, J. C. G. and Nedrum, T., Search for a cardiac nociceptor: Stimulation by bradykinin of sympathetic afferent nerve endings in the heart of the cat, $\mathrm{J}$. Physiol. (Lond.), 306 (1980) 519-536.

3) Blair, R. W., Weber, R. N. and Foreman, R. D., Characteristics of primate spinothalamic tract neurons receiving viscerosomatic convergent inputs in $T_{3}-T_{5}$ segments, J. Neurophysiol., 46 (1981) 797-811.

4) Blair, R. W., Weber, R. N. and Foreman, R. D., Responses of thoracic spinothalamic neurons to intracardiac injection of bradykinin, Circ. Res., 51 (1982) 83-94.

5) Blair, R. W., Weber, R. N. and Foreman, R. D., Responses of spinoreticular and spinothalamic cells to intracardiac bradykinin, Amer. J. Physiol., 246 (Heart Circ. Physiol.) (1984) H500 -H507.

6) Brown, A. M., Excitation of afferent cardiac sympathetic nerve fibres during myocardial is- chemia, J. Physiol. (London), 190 (1967) 35-53.

7) Foreman, R. D., Viscerosomatic convergence onto spinal neurons responding to afferent fibers located in the inferior cardiac nerve, Brain Res., 137 (1977) 167-168.

8) Foreman, R. D. and Weber, R. N., Responses from the cardiopulmonary region and somatic structures, Brain Res., 186 (1980) 463-468.

9) Guzman, R., Braun, C. and Lim, R. K. D., Visceral pain and the pseudoaffective response to intra-arterial injection of bradykinin and other algesic agents, Arch. Int. Pharmacodyn. Ther., 136 (1962) 353-383.

10) Kimura, E., Hashimoto, K., Furukawa, S. and Hayakawa, H., Changes in bradykinin level in coronary sinus blood after experimental occlusion of a coronary artery, Amer. Heart J., 85 (1973) 635-647.

11) Lindgren, I. and Olivecrona, H., Surgical treatment of angina pectoris, J. Neurosurg., 4 (1947) 19-39.

12) Lombardi, F., Della Bella, P., Casati, R. and Malliani, A., Effects of intracoronary administration of bradykinin on the impulse activity of afferent sympathetic unmyelinated fibers with left ventricular ending in the cat, Circ. Res., 48 (1981) 69-75.

13) Nishi, K., Sakanishi, M. and Takenaka, K., Activation of afferent cardiac sympathetic nerve fibers of the cat by pain producing substances and by noxious heat, Pflüger's Arch., 372 (1977) 53-61.

14) Sutton, D. C. and Lueth, H. C., Experimental production of pain on excitation of the heart and great vessels, Arch. Int. Med., 45 (1930) 827867.

15) Taguchi, H., Masuda, T. and Yokota, T., Cardiac sympathetic afferent input onto neurons in nucleus ventralis posterolateralis in cat thalamus, Brain Research, 436 (1987) 240-252.

16) Takahashi, M. and Yokota, T., Convergence of cardiac and cutaneous afferents onto neurons in the dorsal horn of spinal cord in the cat, Neurosci. Lett., 38 (1983) 251-256.

17) Uchida, $Y$. and Murao, S., Excitation of afferent cardiac sympathetic nerve fibers during coronary occlusion, Amer. J. Physiol., 226 (1974) 1094 1099.

18) Uchida, Y. and Murao, S., Bradykinin induced excitation of afferent cardiac sympathetic nerve 
fibers, Jpn. Heart J., 15 (1974) 84-91.

19) Vogt, A., Vetterlein, H., Dal Ri, H. and Schmidt, G., Excitation of afferent fibers in the cardiac sympathetic nerves induced by coronary occlusion and injection of bradykinin, The influence of acetylsalicylic acid and dipyron, Arch. Int. Pharmacodyn. Ther., 78 (1979) 86-98.

20) White, J.C., Cardiac pain, Circulation, 16 (1957) 644-659.

21) White, J. C. and Bland, E. F., The surgical relief of severe angina pectoris: Methods employed and results in 83 patients, Medicine, 27 (1948) $1-42$.

22) White, J. C. and Sweet, W. E., Pain and the Neurosurgeon, Thomas, Springfield, 1969.

Address for correspondence: Toshikatsu Yokota, M. D., Department of Physiology, Medical College of Shiga, Seta, Otsu 520-21, Japan.

Telephone : 0775-48-2147 